\title{
RC Type Cone Broken Motor Structure Statics Analysis and Study of
} Cone

\author{
Chen Yan ${ }^{1, a^{*}}$, Hua Zhongping ${ }^{2, b}$, Chen Jun ${ }^{3, c}$ \\ ${ }^{1}$ Wuhan, Hubei university of technology of Hubei province institute of mechanical engineering \\ ,China \\ ${ }^{2}$ Wuhan, Hubei province of Hubei university of technology institute of mechanical engineering, \\ China \\ ${ }^{3}$ China Railway Siyuan Survey And Design Group Co.,LTD,China \\ a280966494@qq.com, b289586420@qq.com, ㄷ08315499@qq.com
}

Keywords: crusher; moving cone ; FEM

Abstract. this paper takes the RC type hydraulic cone crusher as the research object, using 3D software Solidworks to set up three-dimensional model, and importing it into ANSYS Workbench analysis software to get finite element analysis model, then adding material into mesh model with steps of meshing , loading load and constraint. Structure statics analysis was carried out on the moving cone with the result of deformation and displacement distribution discipline under the effect of load stress. The analysis results show that the design of dynamic cone completely meet the requirements of its strength, stiffness and so on, providing reference data for the structure design.

\section{Introduction}

With the development of industrial technology, the crusher is being constantly updated and improved from the original structure of cone crusher to present hydraulic system which is known as the hydraulic cone crusher ${ }^{[1]}$ adopting hydraulic adjustment to achieve the purpose of discharging mouth and realizing the function of overload protection. Cone crusher is widely used in various field such as metallurgy industry, coal industry, mining industry, construction and chemical industry and so on, due to the prominent advantages of large broken ratio, high efficiency, low energy consumption, laminating crushing and excellent finished product grain shape.It is a most important key processing equipment, a significant component of the "more crushing ${ }^{[2,3]}$ less grinding", and a indispensable device used in two and three stages of crushing system. Moving cone is one of the key components of the cone crusher and its selection and design parameters will directly affect the working life and reliability of the cone crusher. Therefore this paper takes the moving cone of RC type cone broken motor as the research object, analyzing its structure statics based on the software of ANSYS Workbench, providing a theoretical basis ofr the structure designment and improvement.

\section{Linear static analysis}

According to the theory of classical mechanics ${ }^{[4]}$,the universal equation of the dynamics of objects is shown as below:

$$
[M]\left\{\mathrm{x}^{\prime \prime}\right\}+[C]\left\{x^{\prime \prime}\right\}[K]\{x\}=\{F(t)\}
$$

In the equation $[\mathrm{M}]$ denotes mass matrix, $[\mathrm{C}]$ denotes damping matrix, $[\mathrm{K}]$ denotes stiffness matrix, $[\mathrm{x}]$ denotes the displacement vector, $\{\mathrm{F}(\mathrm{t})\}$ denotes a force vector, $\left\{x^{\prime}\right\}$ denotes the velocity vector, and $\left\{\mathrm{x}^{\prime \prime}\right\}$ denotes the acceleration vector.

All the amount related to the time $t$ can be ignored, so the above equation can be written as the following simplified type:

$$
[K]\{\mathrm{x}\}=\{F\}
$$




\section{The working principle and model of cone crusher}

The cone crusher is a complicated model with various component parts, and it mainly consists of cone positioning core parts, brake parts, main shaft parts, eccentric sleeve transmission parts, moving cone parts, hydraulic system, and control system. All parts components are installed on the frame, so the machine frame is just the subject of crusher. In the working process of smashing material of cone crusher, first putting the rocks into the cone crusher at the inlet 1, connecting the right side of transmission shaft to the motor, making the left small bevel gear engaging with the spindle big bevel gear to make the latter turning, then the the big level gear drives eccentric sleeve to rotate and finally force the moving cone to rotate and swimg. The area consists of moving cone 5 and static cone 4 is called the crushing cavity. The material are crushed under multiple extrusion and collision by the pressure of dynamic and static cone. The broken materials meeting the the required size fall to the bottom under the effect of their own gravity when the moving cone deviates from the area and the qualified materials are expelled from the bottom of the cone. The cone crusher achieves continuing material crushing with the continuing and repeated working which satisfy the needs of factory.

The finished part drawings will go through procedures of sub-assembly and final assembly. There are two states under sub-assembly in SolidWorks, respectively fixed state and facile state. The assembly of machine is set to fixed state and the sub-assembly of the rotor and electric machine to facile.

After being assembled, the steps of interference checking and dynamic simulation of sub-assembly are carry out for excluding the interference among different parts which verifying the accuracy and correctness of the designment and correctness and accuracy of calculation. The assembly of cone crusher is show in Fig. 1 as shown below.

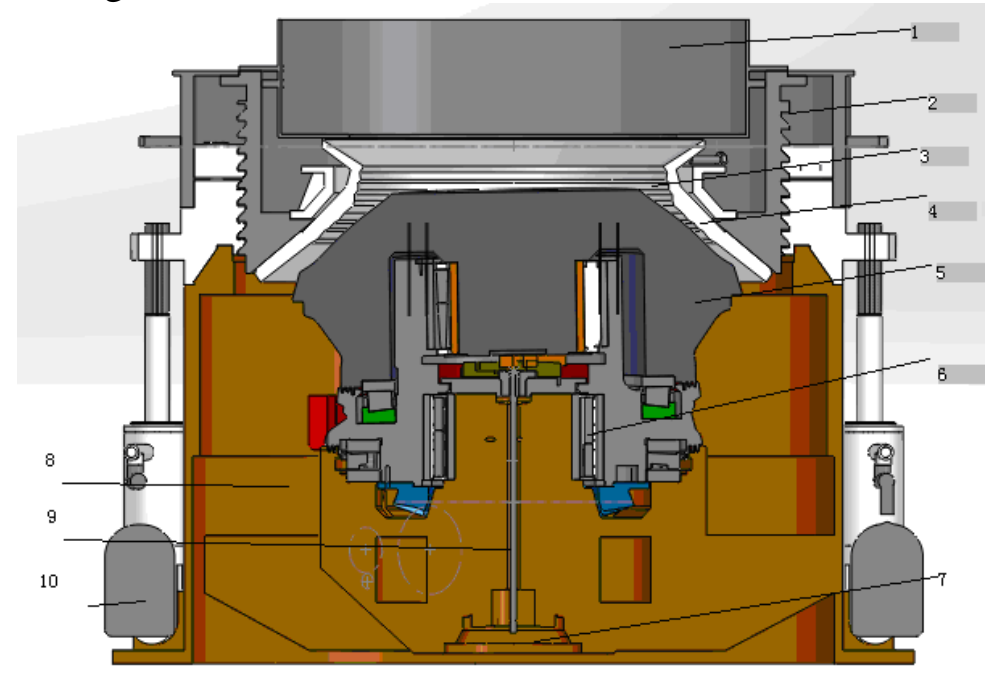

Fig. 1 Cone crusher assembly diagram

As shown in the above Fig. 1,1 is inlet; 2 is adjusting device; 3 is the crushing cavity; 4 is conical parts; 5 is dynamic cone parts; 6 is the rolling bearing; 7 is brake parts; 8 is the frame parts; 9 is spindle; 10 is hydraulic equipment.

\section{The finite element analysis of cone broken mobile cone}

Static analysis of the structure of moving cone is mainly divided into the following steps: importing the geometry model, adding material, adding model material attribute, meshing, computational load and adding constraint conditions. First of all, the 3D model of moving cone 
drawled by $3 \mathrm{~d}$ software are saved as $x$ - $t$ format, and then imported into the finite element software ANSYS Workbench.

\section{Adding the model material attribute}

The first step of static and finite element analysis of cone broken mobile cone is adding material attribute which material is ZGMn13, elastic modulus is $\mathrm{E}=600 \mathrm{Gpa}$, poisson's ratio is 0.28 , mass density is $\rho=7800 \mathrm{~kg} / \mathrm{m}^{3}$, and the dynamic cone material mechanical properties are shown in table 1 .

Table 1 dynamic cone material mechanics performance

\begin{tabular}{cc}
\hline attribute & numerical \\
\hline elasticity modulus/(GPa) & 600 \\
Poisson's ratio & 0.28 \\
modulus of rigidity $/(\mathrm{GPa})$ & 234 \\
mass density $/\left(\mathrm{kg} * \mathrm{~m}^{-3}\right)$ & 7800 \\
tension strength $/(\mathrm{Pa})$ & $7 * 10^{8}$ \\
thermal expansion coefficient $/ \mathrm{k}^{-1}$ & $1.06^{*} 10^{-5}$ \\
thermal conductivity $/\left(W^{*} M^{-1} K^{-1}\right)$ & 14 \\
\hline
\end{tabular}

As shown in Fig. 2 is the moving cone of 3D modeling part drawing:

\section{Meshing}

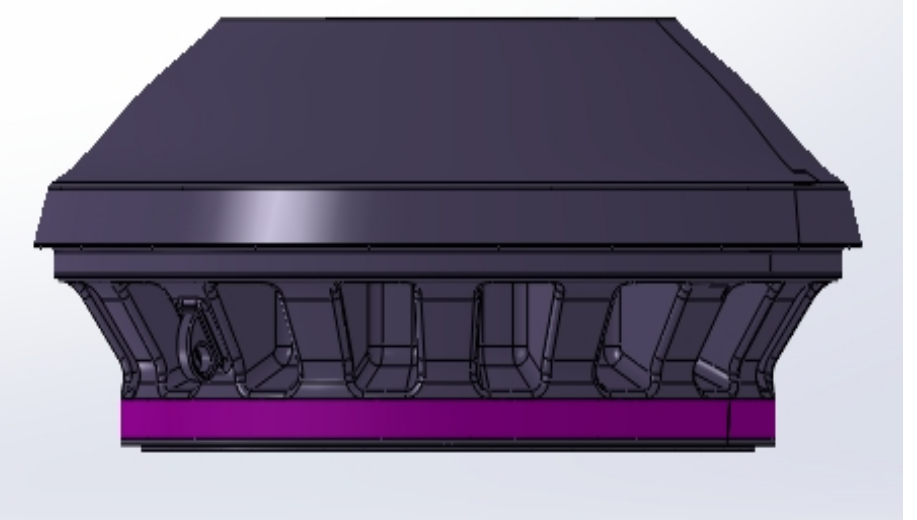

Fig. 2 moving cone $3 \mathrm{~d}$ part drawing

After importing the entity model and simplifying the characteristics of chamfer and rounded corners which have little influence on the results, Medium in the meshing parameters Relevance Center is choosen and the rest stay default preferences .In the process of meshing computing, the moving cone is divided into 102516 units and 177453 nodes. The finite element model of the mobile cone after meshing is shown in Fig. 3.

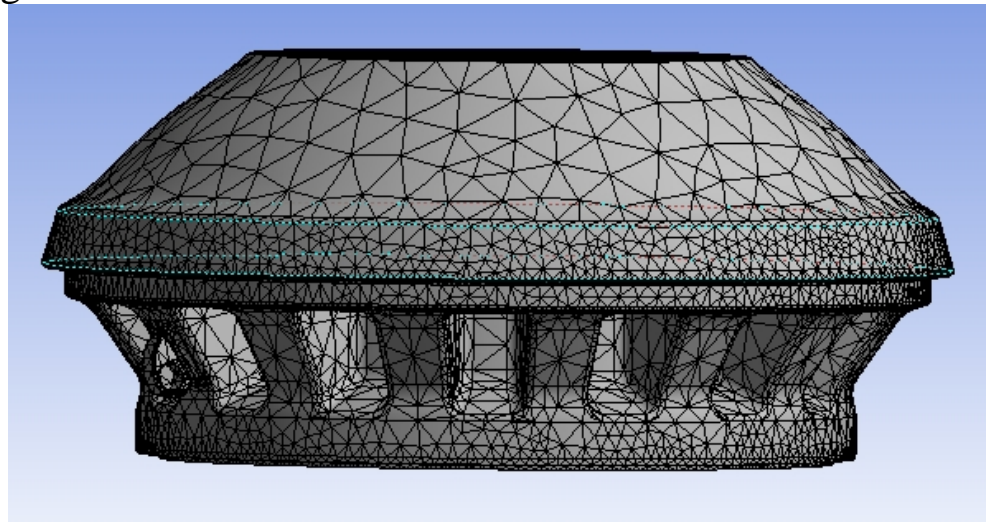

Fig. 3 mesh of moving cone 


\section{Add dynamic cone constraints and load applied \\ Add dynamic cone constraints}

The eccentric shaft sleeve only limits the dynamic cone and thick edge contact point of the radial displacement, not the axial displacement when crushing material. To restrict the turning of the moving cone driven by rolling bearing by the eccentric shaft sleeve, $\mathrm{Y}$ axis rotation constraints is exerted on the bushing bole of moving cone. The bottom of the moving cone is fixed on the eccentric sleeve which stint the axial displacement.

\section{Load calculation and applied}

(1) the unit crushing force along moving cone plate on the vertical direction

In the crushing cavity, the original bulk ore are crushed into small volume in the crusher with gradually increasing from top to the bottom of volume of the loose material. It lead to the gradually decreasing width of crushing cavity and the gap among the material and the gradually increasing degree of compaction of the material from top to bottom. Due to the crushing effect of lining board of the cone, the material is in a tight state, and the gap among the material becomes small which lead to bigger size of the moving cone of lining board of the trip, and the pressure in materials is more and more tight. Therefore, a conclusion ${ }^{[5,6]}$ given by H.Sommer is that on the surface of vertical dynamic cone plate, any section of the unit crushing force is directly proportional to the eccentricity of the corresponding, and inversely proportional to the width of crushing cavity . So unit crushing force along the moving cone plate on the vertical force is in following equation:

$$
\begin{aligned}
& p_{1}=k \frac{e_{y}}{d_{y}} \\
& e_{y}=\frac{y e}{R} \\
& u=(R-y) \tan \alpha
\end{aligned}
$$

Taking Equ.4 and Equ.5 into Equ.3, the unit crushing force formula is produced as follows:

$$
p_{1}=\frac{\mathrm{key} / \mathrm{R} \tan \alpha}{\mathrm{R}+\frac{\mathrm{b}}{\tan \alpha}-y}
$$

$p_{1}$ donate unit crushing force along the moving cone plate in the vertical direction of; $\mathrm{k}$ donate actual situation of the crusher work conversion of value, $\mathrm{k}=11 ; \boldsymbol{e}_{y}$ donate an arbitrary cross section of the eccentricity; $d_{y}$ donate an arbitrary cross section of the crushing cavity width; y donate arbitrary cross section of the unit the position of the crushing force distance, perpendicular to the surface of the moving cone plate; e donate the eccentricity of the cone crusher distance; $\mathrm{R}$ donate the main axle suspension point to the moving cone plate workspace low-end position distance; $\alpha$ donate the meshing angle; $b$ donate the width of the row ore mouth.

(2) The unit crushing force along the circumferential direction of the moving cone plate Due to the roundness of moving cone of lining board and its rotary pendulum movement under the drive of eccentric sleeve, and the scope of work is $-45^{\circ}$ to $45^{\circ}$. The $0^{\circ}$ is the largest stress of location of the middle part. The stress is decreasing along the side, and crushing force is about $0 \mathrm{MPa}$ in the range of $-45^{\circ}$ to $45^{\circ}$. So the decreasing curve of unit crushing force along the moving cone liner circumferential direction can be regarded as parabola.

$$
p_{2}=l x^{2}
$$

(3) unit crushing force effect on the work place of the moving cone plate .

According to last equation the unit crushing force in the work place on the cone plate is: 


$$
p=\frac{k e y / R \tan \alpha}{R+\frac{b}{\tan \alpha}-y}-l x^{2}
$$

\section{The results of post-processing}

The loading crushing force on the cone crusher is $300 \mathrm{MPa}$, the strain figure ,the stress diagram , the total deformation graph of moving cone which is stressed is in the below figure are shown in Fig. 4, Fig.5 and Fig.6.

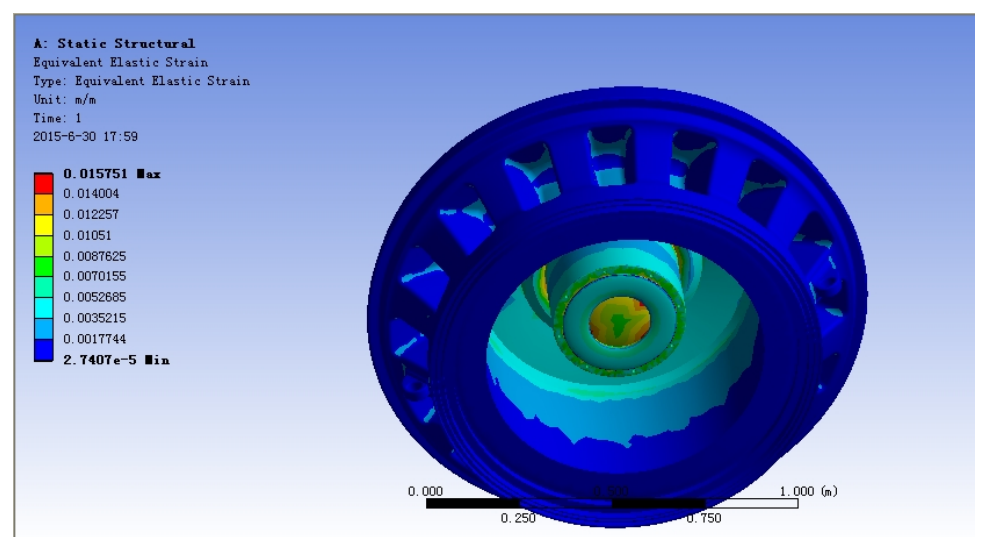

Fig. 4 Cloud moving cone strain analysis

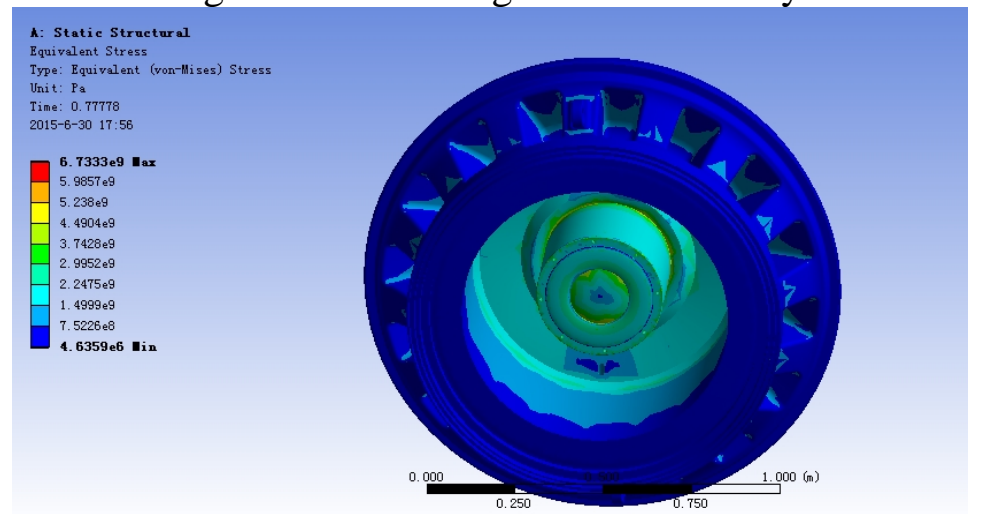

Fig.5 moving cone stress analysis chart

In Fig. 4, the maximum strain value of the moving cone is $0.015751 \mathrm{~m} / \mathrm{m}$ from the stress diagram and the minimum strain value is $2.7407 * 10^{5}$. The strain concentration moving at the corner between the inside and outside of the cone, and the cone and the eccentric sleeve contacting the strain are mainly distributed in the moving cone under the edge nearby.

From the fig. 5 the maximum equivalent stress values of moving cone is $16.7333^{*} 10^{9} \mathrm{pa}$ and the minimum stress $4.6359 * 10^{6}$ pa. The stress concentrated moving between the inside and outside of the cone at corner, because of out of shape by the extrusion of the lining board. The allowed stress of moving cone material is $\left[\sigma_{\mathrm{b}}\right]=240 \mathrm{MPa}$. Diagram of the maximum stress value is far lower than the dynamic of the cone material. The stress value of the contact between the moving cone and the eccentric sleeve is also large, mainly distributed in the upper and lower edge of the moving cone. 


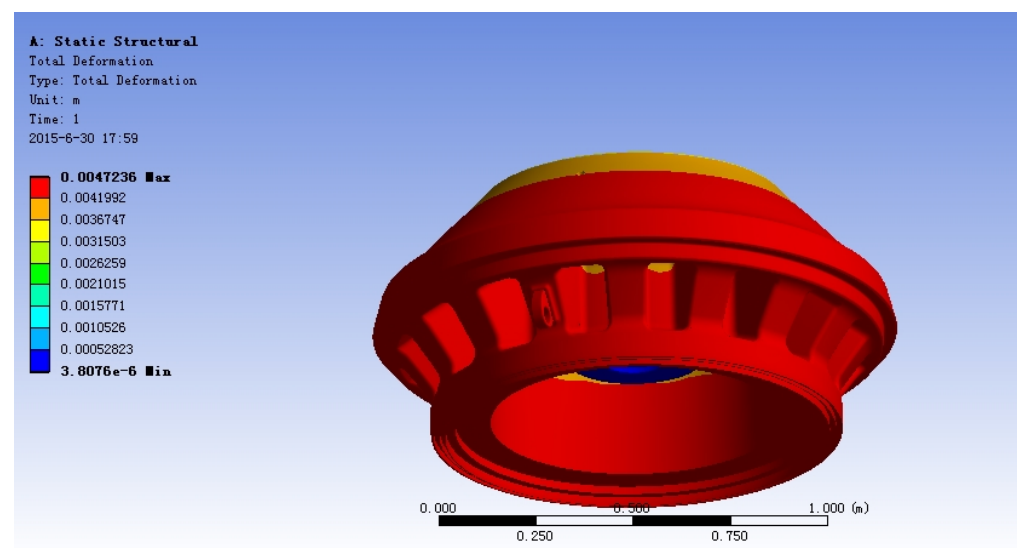

Fig. 6 cloud dynamic cone total deformation analysis

It can be seen that: due to the moving cone driven by the eccentric shaft sleeve and rotary pendulum motion, cooperating with moving cone lining board squeezing materials within the crushing cavity that can achieve crushing the material, the moving cone outer wall displacement deformation is larger, and the lower edge of the moving cone of displacement is larger. The maximal displacement of $0.0047236 \mathrm{~m}$, and the minimum displacement of $3.8076^{*} 10^{-6} \mathrm{~m}$.

\section{Summary}

(1) Moving cone choosing ZGMn13 material conforms to the requirements for the performance of the crusher machine;

(2) The maximum strain value of Moving cone is $0.015751 \mathrm{~m} / \mathrm{m}$, minimum strain value of strain deformation is $2.7407 * 10^{5}$. Strain deformation is concentrated in the inner and outer wall of the moving cone and the contact point of the eccentric sleeve;

(3) The maximum equivalent stress value is $16.7333 * 10^{9} \mathrm{~Pa}$, and the minimum stress is $4.6359 * 10^{6} \mathrm{~Pa}$, which is far less than the allowable stress of the material. The stress concentration is near the edge of the inner wall and the outer wall of the moving cone;

(4) After static analysis, the maximum displacement is $0.0047236 \mathrm{~m}$, the minimum displacement is $\mathrm{m}$, and the maximum deformation position is located at the bottom edge and the outer wall of the moving cone.

\section{References}

[1] Shiping Lang, Baoxian Lang. Crusher[M]Beijing : Metallurgical industry press, 2008, pp.112-113

[2] Changxin Zhang. Short head cone crusher cavity [J]. Industrial Minerals and Processing, 1986.

[3] Baoxian Lang. Cone crusher present situation and developing direction[J]. Mining machinery, 2001, pp. 21-22.

[4] Yanxia Chen , Lei Chen. ANSYS Workbench the engineering application case master[M]Beijing

: Electronic industry press, 2012.

[5] Jiankun Gao. The calculation of finely cone crusher several parameters[J]. Mining machinery, 1991, (5):10-12.

[6] Guangyan Yang, Xichun Min. The finite element analysis of H8800 cone crusher key components[D],Northeastern university, 2011, pp.22-23. 